\title{
DETECTION AND SIGNIFICANCE OF INAPPARENT INFECTION IN CHAGAS DISEASE IN WESTERN VENEZUELA
}

\author{
NESTOR AÑEZ, GLADYS CRISANTE, AGUSTINA ROJAS, HUGO CARRASCO, HENRY PARADA, YANCARLOS YEPEZ, \\ RAFAEL BORGES, PALMIRA GUEVARA, AND JOSE LUIS RAMIREZ \\ Facultad de Ciencias, Instituto de Cardiología, Instituto de Estadística Aplicada y Computación, Universidad de Los Andes, \\ Mérida, Venezuela; Laboratorio de Genética Molecular, Universidad Central de Venezuela, Caracas, Venezuela; \\ Hospital Luis Razetti, Barinas, Venezuela, Universidad Francisco de Miranda, Facultad de Medicina, Coro, Venezuela
}

\begin{abstract}
Inapparent infections of Trypanosoma cruzi were detected in symptomless seropositive people living in close proximity, and under the same conditions of risk, to patients with acute Chagas disease. Similar infections were also detected in sera samples of people from 25 villages of western Venezuela where Chagas disease is endemic. Seropositivity in all the 1,251 studied samples was established by use of 3 serological methods (direct agglutination test, indirect immunofluorescence antibody test, and enzyme-linked immunosorbent assay). Each seropositive sample was tested for detection of anti-T. cruzi-specific immunoglobulin (Ig) $M$ and IgG levels and specific T. cruzi infection by molecular methodology (polymerase chain reaction assay). The combined analysis of the serologic (IgM and IgG levels), molecular (specific T. cruzi DNA), and statistical findings demonstrated the existence of a different stage of $T$. cruzi infection in asymptomatic patients, which is suggested to be recognized as inapparent infection. Its definition, significance, and comparison with typical Chagas disease phases are presented, and its potential epidemiological importance is discussed.
\end{abstract}

\section{INTRODUCTION}

Charles Nicolle ${ }^{1}$ was the first to demonstrate inapparent infections when he discovered that infectious diseases were often accompanied by symptoms that were inapparent to both the practitioner and the patient. The realization that inapparent infections occurred frequently revolutionized ideas about infectious diseases. ${ }^{2}$ This phenomenon was new to epidemiology, and Nicolle used the word "asymptomatic" to contrast this with symptomatic infection. ${ }^{2}$ The author also stressed that the inapparent infection was biologically similar to the patent disease with regard to its natural course, transmissibility, or cure. ${ }^{2}$

Nicolle's concept is now accepted in epidemiology and is closely linked to current ideas about zoonoses. Moreover, it is difficult nowadays to find examples of diseases that are not accompanied by inapparent infections. These include viral, bacterial, and parasitic infections in both animals and humans. ${ }^{2}$ In relation to inapparent infections of Trypanosoma cruzi, Garnham ${ }^{2}$ foresaw its presence and suggested that this stage may be considered in the general context of Chagas disease. He said that although there was no doubt about the clinical manifestation of the disease, he suspected that there were also people with inapparent infection.

Here, we deal with the detection of inapparent infection of $T$. cruzi in symptomless people living in the proximity of acute cases and under the same conditions of risk. In addition, the rate of occurrence of inapparent infections was estimated in samples taken from people who said they had never experienced symptoms attributable to T. cruzi infection. The study was carried out from 1995-1999 on 1,251 human sera samples taken in 25 villages located in 5 states of western Venezuela where Chagas disease is endemic. The diagnosis was made by use of serological criteria to differentiate inapparent infections from acute and chronic cases, and a molecular tool (polymerase chain reaction, or PCR) to corroborate the presence of $T$. cruzi infection.

\section{MATERIALS AND METHODS}

Patients. Two groups of patients were considered; one was made up of 73 asymptomatic people living in close proximity to 14 patients with acute Chagas disease. These acute cases were detected during 1994-1996 as part of a study carried out in areas of western Venezuela where Chagas disease is endemic. ${ }^{3}$ In a previous report, ${ }^{3}$ each acute case was clinically, seroparasitologically, biochemically, and epidemiologically characterized. The asymptomatic group included all the people living in the same area as each detected acute case (Table 1). This group was composed of 44 men $(60.3 \%)$ and 29 women $(39.7 \%)$, with a male to female sex ratio of $1.5: 1$, and a mean \pm standard deviation age of $24.5 \pm 16.3$ years (range, 1-66 years). The second group was made up of 1,178 people from 25 villages of the states of Barinas $(n=194)$, Cojedes $(n=81)$, Falcon $(n=258)$, Merida $(n=192)$, and Portuguesa $(n=453)$ of western Venezuela where Chagas disease is considered to be endemic. ${ }^{3}$ This group was composed of 492 men (41.8\%) and 686 women $(58.2 \%)$ with a male to female sex ratio of $0.7: 1$, and a mean \pm standard deviation age of $27.5 \pm 18$ years (range, 1-87 years).

Written consent from all the patients who entered the protocol or from their representative was obtained before the study plan and sample collection was carried out. The study was approved by the Biomedical Committee of the National Research Council in Venezuela, and the professional medical science committee of the University of Los Andes, Merida, Venezuela.

Sample collection and processing. Samples consisted of peripheral blood obtained by venipuncture from each patient for routine parasitologic and serologic testing. The parasitologic methods used were hemoculture and xenodiagnosis. ${ }^{3}$ The serologic tests used to detect circulating anti-T. cruzi antibodies were a direct agglutination test (DAT) with 2mercaptoethanol, an indirect immunofluorescence antibody test (IFAT), and an enzyme-linked immunosorbent assay (ELISA). Conditions and procedures for serologic and par- 
TABLE 1

Detection of patients with inapparent infection who lived in close proximity to patients with acute Chagas disease*

\begin{tabular}{|c|c|c|c|c|c|c|c|c|}
\hline \multirow[b]{2}{*}{ Code acute } & \multirow{2}{*}{$\begin{array}{l}\text { No. of people } \\
\text { living close to } \\
\text { acute-phase } \\
\text { patients }\end{array}$} & \multicolumn{3}{|c|}{ Serologic diagnosis } & \multicolumn{3}{|c|}{ Ig levels } & \multirow{2}{*}{$\begin{array}{l}\text { Detection of infection } \\
\text { in symptomless } \\
\text { patients }\end{array}$} \\
\hline & & DAT & IFAT & ELISA & $\begin{array}{c}\text { Seropositive, } \\
(\%)\end{array}$ & IgM & $\operatorname{IgG}$ & \\
\hline \multirow[t]{2}{*}{$7-94$} & 9 & - & + & + & $2(22)$ & $1: 512$ & $1: 32$ & Acute \\
\hline & & - & + & + & & $1: 256$ & $1: 32$ & Inapparent \\
\hline $10-94$ & 7 & - & - & - & - & - & - & \\
\hline $11-94$ & 7 & - & - & - & - & - & - & \\
\hline $8-95$ & 6 & + & + & + & $1(16)$ & $1: 256$ & $1: 256$ & Inapparent \\
\hline $20-95$ & 9 & + & + & + & $1(11)$ & $1: 32$ & $1: 256$ & Inapparent \\
\hline \multirow[t]{3}{*}{$10-96$} & 3 & + & + & + & $3(100)$ & $1: 256$ & $1: 1,024$ & Chronic \\
\hline & & + & + & + & & $1: 1,024$ & $1: 256$ & Acute \\
\hline & & + & + & + & & $1: 32$ & $1: 2,048$ & Chronic \\
\hline $12-96$ & 3 & + & + & + & $1(33)$ & $1: 512$ & $1: 128$ & Acute \\
\hline \multirow[t]{4}{*}{$17-96$} & 6 & + & + & + & $4(66)$ & $1: 32$ & $1: 512$ & Chronic \\
\hline & & - & + & + & & $1: 64$ & $1: 64$ & Inapparent \\
\hline & & + & + & + & & $1: 32$ & $1: 128$ & Inapparent \\
\hline & & + & + & + & & $1: 128$ & $1: 16$ & Inapparent \\
\hline $20-96$ & 2 & - & + & + & $1(50)$ & $1: 64$ & $1: 32$ & Inapparent \\
\hline \multirow[t]{2}{*}{$23-96$} & 8 & - & + & + & $2(25)$ & $1: 16$ & $1: 16$ & Inapparent $\dagger$ \\
\hline & & - & + & + & & $1: 128$ & $1: 64$ & Inapparent \\
\hline $24-96$ & 3 & - & + & + & $1(33)$ & $1: 64$ & $1: 32$ & Inapparent \\
\hline $25-96$ & 4 & - & - & - & - & - & - & \\
\hline $26-96$ & 3 & - & - & - & - & - & - & \\
\hline \multirow[t]{2}{*}{$27-96$} & 3 & + & + & + & $2(66)$ & $1: 32$ & $1: 512$ & Chronic \\
\hline & & + & + & + & & $1: 64$ & $1: 16$ & Inapparent \\
\hline Total & 73 & $11(61)$ & 100 & 100 & $18(24.6)$ & & & \\
\hline
\end{tabular}

asitologic methods have been provided elsewhere. ${ }^{3}$ Titers $\geq$ 1:64 for the DAT and IFAT and an optical absorbance $\geq 0.2$ for the ELISA were considered positive for infection by $T$. cruzi. Patients were considered seropositive when they showed reactivity in at least 2 of the 3 serological tests. In addition, samples from seropositive patients were processed for detection of anti-T. cruzi-specific immunoglobulin (Ig) $\mathrm{M}$ and $\mathrm{G}$ levels by means of IFAT. ${ }^{4}$ For quantitation of IgM and IgG levels, we used serum dilution from 1:2 to $1: 4,096$ for each patient exposed to anti-human $\operatorname{IgM}(1: 16)$ and $\operatorname{IgG}$ (1:32) fluorescein isothiocyanate (Sigma Chemical Company, St. Louis, MO).

Diagnostic criteria. To establish the IgM and IgG levels as diagnostic criteria, previous measurements were made with patients with acute and chronic phases of Chagas disease. Clinical, parasitologic, serologic, and molecular status of these patients have been published elsewhere. ${ }^{3,5}$ In both acute- and chronic-phase patients, IgM and IgG levels were determined by the same methodology as indicated above. Acute-phase patients were characterized by high IgM levels, which statistically correspond to titers from 1:512 and up (range, 1:512 to 1:4,096). This contrasted with patients with chronic Chagas disease, who had high IgG levels $(\geq 1: 512)$ and low IgM levels up to $1: 256$ (range, $1: 2$ to $1: 256$ ). The cases were then grouped according to the following criteria. Acute-phase patients were seropositive to $T$. cruzi with high specific anti-T. cruzi $\operatorname{IgM}(\geq 1: 512)$ and low $\operatorname{IgG}$ (up to 1 : 256) levels. Chronic-phase patients were seropositive with low IgM (up to 1:256) and high IgG ( $\geq 1: 512$ ) levels. Symptomless seropositive people had low IgM and low IgG (up to $1: 256)$ levels, and they were considered to have inapparent infection.

Molecular diagnosis. To corroborate the presence of the
T. cruzi genome in those seropositive people considered to have inapparent infections (low IgM and IgG levels), 47 sera samples $(50 \mu \mathrm{L})$ taken at random from the total number of people (111) in this condition were processed by PCR assay. Each $50-\mu \mathrm{L}$ sample was incubated $15 \mathrm{~min}$ at $65^{\circ} \mathrm{C}$ in $1 \times$ Taq polymerase buffer without $\mathrm{Mg}^{++}$and $2 \mu \mathrm{L}$ proteinase $\mathrm{K} 20 \mathrm{mg} / \mathrm{mL}$, followed by incubation at $95^{\circ} \mathrm{C} 15 \mathrm{~min}$. Cellular debris was spun down by centrifugation in an Eppendorf centrifuge for $5 \mathrm{~min}$. Three microliters of this supernatant were used for PCR assay. Conditions for amplification and hybridization have been previously described. ${ }^{5,6}$

Statistical analysis. This consisted of a comparison of PCR as the gold standard test and the DAT, IFAT, and ELISA as alternative tests in people from endemic areas for Chagas disease in western Venezuela. For the analysis, only those samples from people with inapparent infection were considered. The comparison between any methodological pair included estimation of sensitivity, specificity, positive predictive value, negative predictive value, observed agreement, expected agreement by chance, kappa coeficient ${ }^{7}$ with the standard error, $Z$ statistics, and $P$ value. All these values were calculated by Epi Info $6.04 .^{8}$ Pairwise correlation between $\operatorname{IgM}, \operatorname{IgG}$, and age was computed by Kendall's rank correlation tau ${ }^{9}$ with S-Plus statistical software. ${ }^{10}$

\section{RESULTS}

Detection of inapparent infections of $T$. cruzi in close proximity to acute cases of Chagas disease. Serologic tests carried out to discover the status of $T$. cruzi infection in 73 people who lived in close proximity to the 14 selected patients with Chagas disease in its acute phase revealed $24.6 \%$ (18 of 73) seropositivity. The established criteria to quantify 
TABLE 2

Discrimination of clinical conditions based on anti-Trypanosoma cruzi-specific immunoglobulin M and G levels in asymptomatic people from endemic areas of Chagas disease in Venezuela

\begin{tabular}{|c|c|c|c|c|c|}
\hline \multirow[b]{2}{*}{ Venezuelan state } & \multirow[b]{2}{*}{$\begin{array}{c}\text { No. of } \\
\text { sampled people }\end{array}$} & \multirow[b]{2}{*}{$\underset{(\%)}{\text { Seropositive, } n} n$} & \multicolumn{3}{|c|}{ Clinical condition* } \\
\hline & & & $\begin{array}{l}\text { Acute, } n \\
(\%)\end{array}$ & $\begin{array}{c}\text { Chronic, } n \\
\quad(\%)\end{array}$ & $\underset{(\%)}{\text { Inapparent, } n}$ \\
\hline Barinas & 194 & $50(25.7)$ & $5(10)$ & $15(30)$ & $30(60)$ \\
\hline Cojedes & 81 & $25(30.8)$ & $1(4)$ & $18(72)$ & $6(24)$ \\
\hline Falcon & 258 & $4(1.5)$ & - & $3(75)$ & $1(25)$ \\
\hline Merida & 192 & $14(7.3)$ & - & $6(42.8)$ & $8(57.1)$ \\
\hline Portuguesa & 453 & $88(19.4)$ & - & $22(25)$ & $66(75)$ \\
\hline Total & 1,178 & $181(15.3)$ & $6(3.3)$ & $64(35.4)$ & $111(61.3)$ \\
\hline
\end{tabular}

and low IgG. See the text for level definitions.

$\operatorname{IgM}$ and $\operatorname{IgG}$ levels allowed us to detect from this asymptomatic group 3 acute (16.7\%), 4 chronic (22.2\%), and 11 $(61.1 \%)$ inapparent infections. Details of the proportion of infected people detected and the respective $\mathrm{IgM}$ and $\mathrm{IgG}$ levels of the symptomless people are shown in Table 1. No blood-circulating trypomastigotes of $T$. cruzi were detected in any patient when xenodiagnosis and hemoculture were performed.

Estimation of inapparent infection of Trypanosoma cruzi in areas endemic for Chagas disease in Venezuela. From 1,178 people sampled in endemic areas, 181 (15.3\%) were seropositive for $T$. cruzi. The quantification of anti-T. cruzi specific Ig titers revealed people with asymptomatic acute infections (3.3\%) and others in the chronic stage $(35.4 \%)$ of Chagas disease, judging by their high IgM and IgG levels, respectively. Similarly, the presence of inapparent infection was shown in $111(61.3 \%)$ people who claimed that they had never experienced any clinical symptoms attributable to $T$. cruzi infection, and in whom low levels of specific $\operatorname{IgM}$ and $\operatorname{IgG}$ (from 1:2 to $1: 256$ ) were detected. Table 2 gives details of the estimation of clinical condition on the basis of the measurements of specific IgM and IgG levels in patients from 5 states of western Venezuela. The frequency distribution according to Ig level is shown in Table 3 .

Molecular findings in people with inapparent infections. We carried out a $T$. cruzi-specific PCR assay in sera samples of 47 people with inapparent infection. This assay amplified $130 \mathrm{bp}$ within the ribosomal spacer of the parasite. Figure 1 shows some of the positive samples, which showed a strong PCR signal with the expected band size, whereas the negative control showed no signal at all.
Statistical analysis. Statistical analysis showed that serological methods were very reliable, as indicated by the high level of concordance between the methods $(>0.85)$, high sensitivity (0.80-0.93), and the high specificity (100), as well as the substantial agreement when compared with PCR assay as a gold standard test ( $\kappa, 0.66-0.85 ; Z, 5.35-$ 6.76; $P<0.0001$ ). In Table 4 , details are provided of the results obtained when serological techniques, (DAT, IFAT, and ELISA) used as alternative tests were compared with the PCR assay (the gold standard test). According to Kendall's rank correlation tau, no statistical significance was observed between the IgM and IgG levels and the IgM level and the age of infected people $(P>0.50)$. However, the same analysis revealed a high correlation between $\operatorname{IgG}$ level and the age of infected people $(P<0.001)$.

\section{DISCUSSION}

In the present study, 1,251 people-73 living in close proximity to patients with acute Chagas disease and 1,178 from rural villages of western Venezuela-were serologically examined to detect circulating anti- $T$. cruzi antibodies. From the total samples tested, 199 (15.9\%) satisfied the established criteria to be considered positive to $T$. cruzi infection-that is, the coincidence of the 3 serologic methods used for diagnosis. The high confidence level provided by statistical analysis of the results suggests that seropositive people have had previous contact with $T$. cruzi. However, according to their own testimony at the time of sampling, they had never exhibited symptoms attributable to Chagas disease, suggesting the presence of inapparent or occult in-

TABLE 3

Frequency distribution of clinical condition of patients with Chagas disease from different localities according to immunoglobulin (Ig) levels

\begin{tabular}{|c|c|c|c|c|c|c|c|c|}
\hline \multirow[b]{2}{*}{$\operatorname{IgM}$} & \multicolumn{8}{|c|}{$\operatorname{IgG}$} \\
\hline & $1: 16$ & $1: 32$ & $1: 64$ & $1: 128$ & $1: 256$ & $1: 512$ & $1: 1,024$ & $1: 2,048$ \\
\hline $1: 8$ & 0 & 0 & 0 & 0 & 0 & 1 & 0 & 0 \\
\hline $1: 16$ & 6 & 7 & 8 & 6 & 4 & 6 & 6 & 0 \\
\hline $1: 32$ & 3 & 8 & 8 & 14 & 7 & 19 & 10 & 1 \\
\hline $1: 64$ & 4 & 7 & 4 & 4 & 6 & 7 & 8 & 0 \\
\hline $1: 128$ & 4 & 3 & 0 & 3 & 3 & 5 & 1 & 0 \\
\hline $1: 256$ & 0 & 0 & 1 & 0 & 1 & 0 & 0 & 0 \\
\hline $1: 512$ & 0 & 1 & 1 & 0 & 0 & $1 *$ & 0 & 0 \\
\hline $1: 1,024$ & 0 & 1 & 1 & 0 & 0 & $1 *$ & 0 & 0 \\
\hline
\end{tabular}

* These patients were classified as late acute-phase cases. 


\section{A}

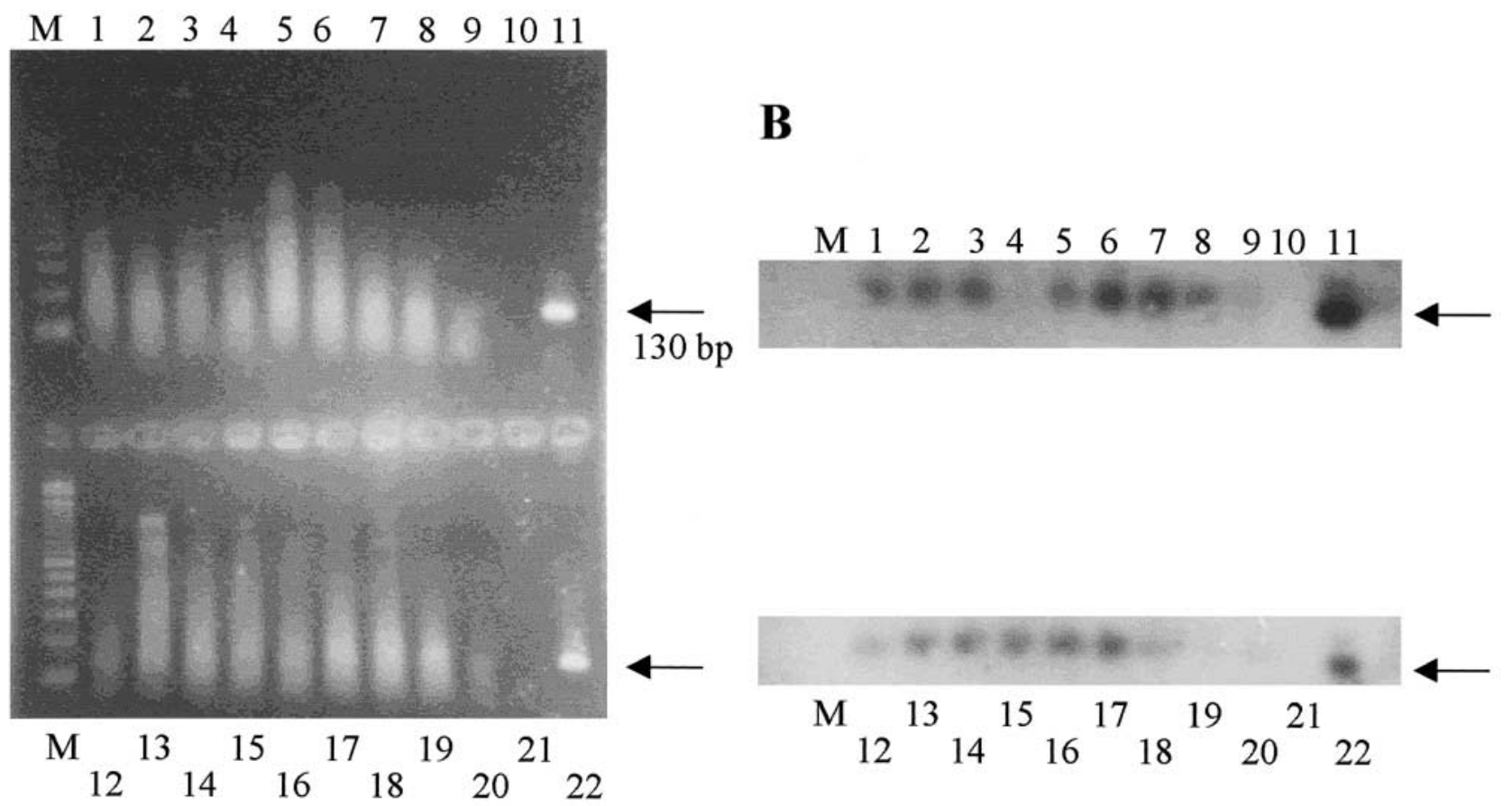

FIGURE 1. Trypanosoma cruzi species-specific polymerase chain reaction (PCR) assay in sera samples of patients with inapparent infection from endemic areas for Chagas disease in western Venezuela. A. Ethidium bromide-stained gel showing PCR amplification products. Lanes 1-8 and 12-16 indicate sera samples from people with inapparent infection. Lanes 9 and 19 indicate sera from noninfected people, which were used as negative controls. Lanes 11 and 22 indicate the product of PCR assay on T. cruzi-purified DNA used as positive control. Lane 17 indicates serum from an acute-phase patient with Chagas disease. Lane 18 indicates serum from a chronic-phase patient with Chagas disease. Lane 20 indicates negative control for reagents. $\mathbf{M}=$ molecular weight marker. B. Southern blot transfer of gel shown in $\mathbf{A}$ after hybridization with a $T$. cruzi-specific probe.

fections. These findings support previous statements that most people with $T$. cruzi infection are asymptomatic. ${ }^{11}$

We also insist on the importance of distinguishing between patients with real inapparent Chagas disease and those who were oligosymptomatic or who had minimal symptomatology. In this regard, the quantification of anti-T. cruzispecific IgM and IgG levels in the 199 seropositive and symptomless patients allowed us to group them into 3 categories, as follows. First are patients with high $\operatorname{IgM}$ and low IgG levels, representing $4.5 \%$ (9 of 199) of the seropositive group. This group had IgM levels similar to those found in 59 confirmed acute chagasic cases, previously reported in the same study region, ${ }^{3}$ and to those reported from endemic areas in Bolivia. ${ }^{12}$ These are the kind of patients who, despite having blood-circulating parasites, do not show any symptoms attributable to $T$. cruzi infection. ${ }^{3,13}$ This fact on the one hand may suggest the possibility of indoor human-tohuman transmission when domiciliar triatomine insects are present, and on the other hand, may lead us to consider asymptomatic infected people as potential intradomiciliar $T$. cruzi reservoirs. Second is the group of patients comprising 68 asymptomatic people (34.2\%) with high IgG but low IgM levels. These levels were similar to those detected in a group of chronic patients who were followed up from the time they

TABLE 4

Comparison of diagnostic methods in people with inapparent infection using polymerase chain reaction as the gold standard*

\begin{tabular}{|c|c|c|c|}
\hline \multirow[b]{2}{*}{ Variable } & \multicolumn{3}{|c|}{ Alternative test } \\
\hline & DAT & IFAT & ELISA \\
\hline Sensitivity (95\% CI) & $80.5(64.9-90.6)$ & $85.4(70.1-93.9)$ & $92.7(79-98.1)$ \\
\hline Specificity (95\% CI) & $100(71.1-100)$ & $100(71.1-100)$ & $100(71.1-100)$ \\
\hline Positive predictive value $(95 \% \mathrm{CI})$ & $100(87-100)$ & $100(87.7-100)$ & $100(88.6-100)$ \\
\hline Negative predictive value (95\% CI) & $61.9(38.7-81)$ & $68.4(43.5-86)$ & $81.3(53.7-95)$ \\
\hline Observed concordance & 0.851852 & 0.888889 & $(0.944444)$ \\
\hline Expected concordance by chance & 0.557613 & 0.576816 & $(0.605624)$ \\
\hline Kappa coefficient (standard error of $\kappa$ ) & $0.666116(0.128225)$ & $0.737439(0.131308)$ & $0.859130(0.134726)$ \\
\hline$Z$ & 5.19 & 5.62 & 6.38 \\
\hline$P$ value & 0.0 & 0.0 & 0.0 \\
\hline
\end{tabular}

* Acute- and chronic-phase patients were excluded for this analysis. CI = confidence interval; DAT $=$ direct agglutination test; ELISA $=$ enzyme-linked immunosorbent assay; IFAT $=$ indirect immunofluorescence antibody test. 
were in the acute phase. ${ }^{5}$ This fact reveals that asymptomatic people with the serologic profile characteristic of chronic chagasic patients appear to be more frequent than previously believed, reaching one third of the detected seropositive population in our study. Third are those patients with low IgM and low IgG levels, representing the highest proportion (61.3\%) of the 199 people that resulted seropositive for $T$. cruzi. The $T$. cruzi-infected people (or in the case of infected children, their representatives) denied ever having experienced clinical symptoms associated with Chagas disease before they were sampled.

Information gathered in the present work makes it possible to identify people exposed to risk conditions in chagasic areas who are asymptomatic from the clinical point of view, and who test negative in parasitologic examination but are seropositive for $T$. cruzi with low anti-T. cruzi-specific IgM and IgG levels (as with inapparent infections), representing those people identified as healthy carriers. For a high confidence level in identifying an inapparent infection in a patient, this must be demonstrable by a much more specific and sensitive methodology such as PCR, as in the present study, with 47 randomly chosen samples.

The above makes it possible to distinguish the typical described phases of Chagas disease in symptomless people and to distinguish them from inapparent infection. This includes the following. 1) Acute-phase asymptomatic patients, characterized by seropositivity to $T$. cruzi and the presence of high IgM and low IgG levels. These patients are obviously experiencing a recent infection and need to be treated to avoid further clinical complications. 2) Chronic-phase asymptomatic patients, seropositive to $T$. cruzi, with high specific IgG and low IgM levels. These patients have experienced and overcame an occult acute episode and need to be checked by methods other than electrocardiogram, which is unable to detect cardiac problems at this stage. ${ }^{5}$ 3) Real inapparent infection. This is detected in symptomless people when serological diagnosis gives positive results to $T$. cruzi accompanied by low IgM and IgG levels.

Obviously, the stages for Chagas disease described above in symptomless people reveal a well balanced host-parasite relationship, which may lead to confusion if the analysis is not sufficiently sensitive. However, when anti-T. cruzi-specific Ig levels are examined, it is possible to discriminate between these asymptomatic forms, as described above. This differentiation is particularly important in that the higher the IgM levels, the nearer the cause of the infection. This information would be useful to detect recently infected people and to control the mode of transmission both by vector and blood transfusion.

The results given in the present work demonstrating different phases of Chagas disease in asymptomatic people have added to the recent proposal of Añez and coworkers ${ }^{5}$ that the so-called indeterminate phase of the infection has no justification because of the constant parasite persistence and myocarditis found in chronic chagasic patients. This fact leads us to divide the course of the chagasic infection into 3 phases: acute, chronic, and inapparent. The acute phase may take 2 forms: the typical symptomatic form with different clinical symptoms, ${ }^{3}$ positive to parasitologic tests, frequently showing blood-circulating parasites, positive to serological tests, and with high IgM levels. Histopathologic methods reveal acute myocarditis, ${ }^{14}$ and immunohistochemical techniques show antigen deposits in the myocardial fiber and the presence of $T$. cruzi amastigotes. ${ }^{5}$ Specific PCR assay gives positive results both in serum and endomyocardial biopsy of the patient. ${ }^{5}$ On the other hand, acute asymptomatic form gives no apparent clinical manifestation, and would be negative for parasite tests but seropositive, showing high IgM and low IgG levels. The chronic phase also has 2 forms. The typical symptomatic form shows clinical manifestation, ${ }^{15}$ is frequently negative to parasitologic tests, and is seropositive, showing high IgG levels. Histopathologic examination reveals chronic myocarditis with necrosis, inflammatory infiltrate, and fibrosis at different levels, and histochemical methods show antigenic deposits and parasites in the heart fibers. In addition, PCR is positive for sera and biopsy samples. ${ }^{5}$ The asymptomatic chronic forms have no apparent clinical manifestation and are negative to parasitologic tests but seropositive to $T$. cruzi, with high IgG and low IgM levels. Finally, there is the inapparent phase, characterized by no apparent clinical manifestation and negative for circulating parasites, but seropositive for anti-T. cruzi antibodies, with low levels of specific IgM and IgG, but PCR positive. This kind of infection must be seriously taken into consideration because of its potential epidemiological importance, as demonstrated by the high proportion of detected cases both in the proximity of acute cases and in that part of the population living in endemic areas under high-risk conditions.

There is little information in the literature about inapparent or occult infections. However, this kind of infection has been either suggested for other infectious diseases from the early $1900 \mathrm{~s}^{1}$ or recently demonstrated for Chagas disease in areas of the neotropical region. . $^{3,12,13}$

Although at present no definitive explanation can be given for inapparent infection in people living in areas endemic for Chagas disease, some possibilities may be suggested. These include the following: low numbers of T. cruzi metacyclic infective forms deposited by infected triatomine insects, which, having reached the host cells, is followed by slow multiplication. This enables the immunity mechanism of the host to destroy part of the parasite population, establishing a host-parasite balance, making it possible for the infected individual to show no symptoms, which is recognized as inapparent infection. Another possibility could be a previous contact with a parasite that shares antigen components with $T$. cruzi. This would be the case of $T$. rangeli, a species that circulates in the same endemic areas, has the same geographical distribution, and shares the same vectors and reservoirs as $T$. cruzi. ${ }^{16,17}$

Whatever the reason for the presence of inapparent infections in the population of endemic areas for Chagas disease, what is important is to know that people with these inapparent infections will eventually develop the disease in a severe form, as previously suggested. ${ }^{18}$ This fact has been demonstrated in the present work by the conversion or reactivation of a patient with inapparent infection into symptomatic infection 1-3 months after being diagnosed (see Table 1). Our results also support Garnham's assumption that "inapparent infections are at least as numerous as and probably much in excess of the clinical disease." ${ }^{18}$ The fact of 
finding $61 \%$ T. cruzi inapparent infections appears to corroborate this statement.

Finally, as emphasized by Nicolle, ${ }^{1}$ it is important to estimate to what degree inapparent infections constitute a reservoir and how these infections affect young children, which could act as a potential genesis of an epidemic.

Acknowledgments: We are indebted to Prof. I. Woodward for careful review of the manuscript; Dr. Pablo Garcia for his suggestions; and Marcos Aguilera, Néstor Añez, Jr., and Nelly Paredes for technical assistance.

Financial support: This work was supported by Consejo de Desarrollo Científico, Humanístico y Tecnológico, Universidad de Los Andes, Mérida, Venezuela, grant CDCHT-ULA-C-743-95-AA (N.A.) and CONICIT grants G-99000036 (N.A.), S1-95000524 (J.L.R.) and RLA 06-26 IAAE (P.G.)

Authors' addresses: Néstor Añez, Gladys Crisante, and Agustina Rojas, Universidad de Los Andes, Facultad de Ciencias, Departamento de Biología, Mérida, 5101, Venezuela. Hugo Carrasco, Instituto de Cardiología, Hospital Universitario de Los Andes, Mérida, 5101, Venezuela. Henry Parada, Unidad de Cardiología, Hospital Luis Razetti, Barinas, Venezuela. Yancarlos Yepez, Universidad Francisco de Miranda, Facultad de Medicina, Coro, Venezuela. Rafael Borges, Instituto de Estadística Aplicada y Computación, Facultad de Economía, Universidad de Los Andes, Mérida, Venezuela. Palmira Guevara and José Luis Ramirez, Grupo de Genética Molecular, Facultad de Ciencias, Universidad Central de Venezuela, Caracas, Venezuela.

Reprint requests: Néstor Añez, Universidad de Los Andes, Facultad de Ciencias, Departamento de Biología, Mérida, 5101, Venezuela (e-mail: nanes@ ciens.ula.ve).

\section{REFERENCES}

1. Nicolle C, 1933. Les infections inapparentes. Scientia 33: 181271.

2. Garnham PCC, 1977. Charles Nicolle and inapparent infections. Am J Trop Med Hyg 26: 1101-1104.

3. Añez N, Carrasco H, Parada H, Crisante G, Rojas A, Gonzalez N, Ramirez JL, Guevara P, Rivero C, Borges R, Scorza JV, 1999. Acute Chagas' disease in western Venezuela: a clinical, seroparasitologic, and epidemiologic study. Am J Trop Med Hyg 60: 215-222.

4. Camargo ME, 1966. Fluorescent antibody test for serodiagnosis. Technical modification employing preserved culture forms of
Trypanosoma cruzi in a slide test. Rev Inst Med Trop Sao Paulo 8: 227-234.

5. Añez N, Carrasco H, Parada H, Crisante G, Rojas A, Fuenmayor C, Gonzalez N, Percoco G, Borges R, Guevara P, Ramirez JL, 1999. Myocardial parasite persistence in chronic chagasic patients. Am J Trop Med Hyg 60: 726-732.

6. Novak EM, DeMello MP, Gomez HBM, Galindo I, Guevara P, Ramirez JL, Da Silveira JF, 1993. Repetitive sequences in ribosomal intergenic spacer of Trypanosoma cruzi. Mol Biochem Parasitol 60: 273-280.

7. Cohen J, 1960. A coefficient of agreement for nominal scale. Educ Psychol Meas 20: 37-46.

8. Dean AG, Dean JA, Coulombier D, Brendel KA, Smith DC, Burton AH, Dicker RC, Sullivan K, Fagan RF, Arner TG, 1995. Epi Info, Version 6: A Word-Processing, Database, and Statistical Program for Public Health on IBM-Compatible Microcomputers. Atlanta: Centers for Disease Control and Prevention.

9. Stuart A, Ord JK, 1991. Kendall's Advanced Theory of Statistics. Vol. 2. Oxford: Oxford University Press.

10. Mathsoft, 1999. S-Plus 2000 Guide to Statistics. Vol. 1. Seattle: Mathsoft.

11. Lugones H, Ledesma O, Storino R, Marteleur A, Meneclier CR, Barbieri G, 1994. Chagas agudo. Storino R, Milei J, eds. Enfermedad de Chagas. Doyma, Argentina: Mosby, 209-233.

12. Medrano-Mercado N, Luz MRPM, Torrico F, Tapia G, Van Leuven F, Araujo-Jorge TC, 1996. Acute phase proteins and serological profiles of chagasic children from an endemic area in Bolivia. Am J Trop Med Hyg 54: 154-161.

13. Rassi A, Luqueti AD, Rassi A Jr, Rassi SG, Rassi AG, 1992. Chagas' disease: clinical features. Wendel S, Brener Z, Camargo W, Rassi A, eds. Chagas' Disease (American trypanosomiasis): Its Impact on Transfusion and Clinical Medicine. Sao Paulo: ISBT Brazil 1992, 81-101.

14. Parada H, Carrasco H, Añez N, Fuenmayor C, Inglessis I, 1997. Cardiac involvement is a constant finding in acute Chagas' disease: a clinical, parasitological and histopathological study. Int J Cardiol 60: 49-54.

15. Carrasco H, Parada H, Guerrero L, Duque M, Duran D, Molina C, 1994. Prognostic implications of clinical, electrocardiographic and hemodynamic findings in chronic Chagas disease. Int J Cardiol 43: 27-28.

16. D'Alessandro-Bacigalupo A, Saravia N, 1992. Trypanosoma rangeli. New York: Academic Press.

17. Añez N, 1982. Studies on Trypanosoma rangeli TEJERA, 1920. IV. A reconsideration of its systematic position. Mem Inst Oswaldo Cruz 77: 405-415.

18. Garnham PCC, 1980. The significance of inapparent infections in Chagas' disease and other forms of trypanosomiasis. Mem Inst Oswaldo Cruz 75: 181-188. 